\title{
Tanguy Viel : imaginaires d'un romancier
}

\author{
Roger-Michel Allemand \\ Laboratoire Babel - Université du Sud Toulon - Var
}

Roger-Michel Allemand - De mai à juillet derniers, le Grand Palais a consacré une rétrospective à la figuration narrative. Vous avez été beaucoup sollicité à cette occasion. Pourquoi ?

Tanguy Viel - J'ai rencontré Jacques Monory il y a quelques années pour un travail de commande qu'on nous proposait en commun. Nous avons très vite sympathisé et sommes devenus, je crois, assez complices dans nos vues. Et puis sa peinture, c'est vrai, a plusieurs points communs avec ce que j'essaye de faire, notamment l'utilisation du cinéma et plus généralement le recyclage des images, mêlé à des sources plus autobiographiques : c'est ce mixage qui est souvent très beau. Alors voilà, c'est un peu le hasard aussi, mais du coup j'ai 
accepté de réfléchir un peu sur ce que pouvait être la figuration narrative. Mais pour tout vous dire, en dehors des œuvres de Monory et de Aillaud, j'ai été plutôt déçu.

R.-M. A. - Ancré dans l'imagerie populaire de la société de consommation, ce mouvement tentait de rompre avec l'abstraction. Qu'en pensez-vous?

T. V. - En réalité, je ne me sens pas tellement proche de la figuration narrative en tant que mouvement, justement parce qu'elle est très ancrée historiquement, et même souvent revancharde à l'égard des autres mouvements de son époque. Tout ce qui, dans la figuration narrative, a été fait dans un sens polémique et politique a produit une peinture volontariste qui vieillit plutôt mal.

R.-M. A. - Circonscrit aux Trente glorieuses, elle a pâti de l'hégémonie du pop art.

T. V. - Le pop art est plus intelligent. Il a plus d'humour sur lui-même. Il a un dandysme que n'auront jamais atteint les peintres français. J'excepte Monory bien sûr, qui a de la classe.

R.-M. A. - En France, la régénération picturale est également passée par les nouveaux réalistes, rejetés par la figuration narrative au point que dans l'ensemble provocateur Vivre et laisser mourir ou la fin tragique de Marcel Duchamp, en octobre 1965, le huitième et dernier tableau montre le cercueil du peintre, drapé dans la bannière américaine, porté par trois artistes pop new yorkais et par trois nouveaux réalistes parisiens, ainsi désignés comme repoussoirs. 

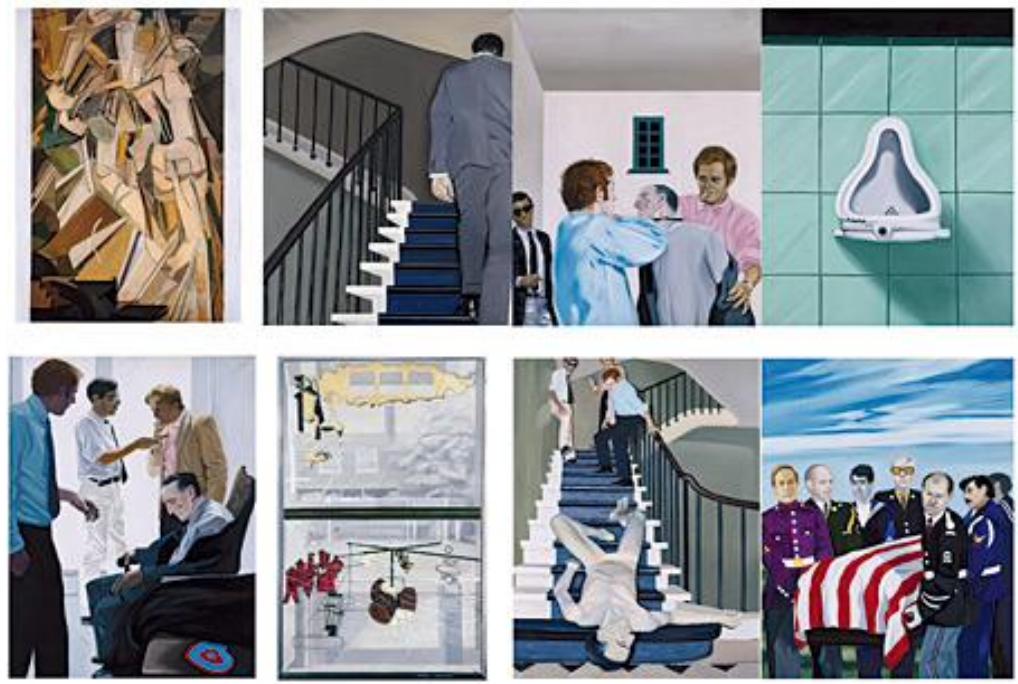

Figure 1. "Vaincre et laisser mourir ou la fin tragique de Marcel Duchamp », une œuvre en huit tableaux de Gilles Aillaud, Eduardo Arroyo et Antonio Recalcati, 1965. - Photo : ADAGP, Paris, 2008.

T. V. - J'avoue que je n'ai jamais compris la violence du conflit avec les nouveaux réalistes, encore moins le sens trop grossier de ces tableaux douteux. Il aurait pu y avoir beaucoup plus de relations entre les deux mouvements et surtout beaucoup plus de porosité qui n'aurait pas desservi la figuration narrative, au contraire. J'ai entendu il y a encore deux mois Rancillac nous expliquer que Duchamp était un escroc. Ce genre de discours en 2008, vous serez d'accord avec moi que c'est vraiment stupide. Dans ces conditions, il est difficile de bâtir un dialogue, mais encore plus une œuvre solide.

R.-M. A. - Duchamp assassiné, c'est bien entendu la mise à mort de l'art conceptuel. Comment vous situez-vous dans ce débat, que ce soit en termes picturaux ou littéraires? Les deux domaines sont-ils comparables? 
T. V. - J'entretiens avec Duchamp la même proximité qu'avec Maurice Blanchot ou Samuel Beckett, c'est-à-dire une admiration sans borne. Ils sont ceux qui ont touché au nœud de la création. Il ne s'agit pas de faire comme eux bien sûr puisqu'ils ont épuisé leur propre sillon, mais je n'arrive pas à comprendre comment on peut être artiste, toute discipline confondue, et ne pas se reconnaître dans ces gestes-là, d'extrême ironie, d'extrême fatigue ou d'extrême désespoir. Du coup, je ne fais pas une différence aussi radicale, dans le geste de créer, entre une tentative purement conceptuelle et, à l'inverse, une hypertrophie de la figuration. Ce sont deux postures inverses mais qui devraient être capables de se reconnaître mutuellement comme sœurs. En tout cas dans l'écriture, ce sont deux états très voisins qui peuvent mener de l'assèchement pur aux images les plus débridées. La différence ensuite entre écrire et peindre, c'est qu'écrire ne peut jamais être un geste conceptuel : paradoxalement, le langage produit plus forcément de l'image que la peinture. De là que nous n'avons jamais eu d'une manière aussi virulente ce débat entre figuration et abstraction. Et parce que devant une phrase, je suis sûr que Samuel Beckett et Alexandre Dumas, pour prendre les deux extrêmes, peuvent ressentir la même chose.

R.-M. A. - Vous avez en tout cas au moins un point commun avec les tenants de la figuration narrative, du point de vue de l'arrière-plan culturel: la plupart de ces raconteurs d'histoires fréquentaient la Cinémathèque et empruntèrent volontiers des formes à la Nouvelle Vague. Chez vous, dès Le Black Note, on observe une écriture quasi cinématographique, dans les images, les plans-séquences et le montage. Qu'est-ce que votre cinéphilie a précisément apporté à votre manière d'aborder un récit? 
T. V. - En premier lieu, je crois, un capital d'images disponibles: le cinéma a formalisé des archétypes, à la fois comme aboutissement de la littérature passée, à la fois comme renouvellement de ces figures actualisées dans le monde moderne en tant que plus urbain, plus mouvementé, plus nocturne, etc. C'est donc une mémoire et donc un matériau pour moi, au même titre que l'enfance ou la réalité perçue. Mais bien sûr le cinéma est aussi une forme, et en tant que telle, elle « préfigure » ou « configure » notre cerveau. De ce point de vue, je crois que l'apport le plus important sur mon propre travail est celui du rythme, c'est-à-dire de l'enchaînement des durées. Par exemple, j'ai l'impression de penser mes livres sur deux heures maximum, avec des pleins et des creux qui ont l'air d'être inconsciemment calqués sur un rythme de narration cinématographique. Mais ce rythme du cinéma emprunte luimême à des formes très archaïques, souvent proches du théâtre grec. Donc on pourrait dire les choses dans l'autre sens : que c'est toujours la littérature, en tant qu'art du récit, qui tient les ficelles.

R.-M. A. - Cinéma est construit à partir du dernier film de Joseph Mankiewicz, Le Limier. En quoi, dès lors, a consisté votre travail d'écrivain?

T. V. - Ce travail a eu lieu à une époque où le cinéma possédait pour moi une puissance narrative et notamment mimétique, que je ne me sentais pas capable d'égaler par la phrase. Alors c'était comme un jeu d'impuissance que de plier le genou devant le cinéma en disant: il ne reste que ça à faire, essayer de raconter un film. À ce point qu'au départ, le projet était très froid, très formel, consistant seulement à décrire cliniquement l'objet. Mais très vite, ma propre phrase achoppait 
contre les images et c'est curieusement de cet achoppement qu'est né le narrateur. C'est seulement là que le livre a pu avoir du sens pour moi, quand j'ai compris que l'enjeu n'était pas seulement le «concept» du remake littéraire, mais la scène balbutiante et obsessionnelle du discours. De sorte qu'à la fin, je crois, c'est plus un livre sur la cinéphilie que sur le cinéma.

R.-M. A. - Vous vous êtes beaucoup intéressé au polar et aux films de série B; l'intrigue et la structuration de vos romans sont communément rattachées au genre policier. D'où vient cet attrait et sur quoi repose-t-il?

T. V. - Je me suis longtemps posé cette question, surtout que je ne lis pas du tout de romans policiers. Ma première réponse fut souvent de croire qu'il y avait là, sur la scène criminelle, une densité de passions et de sentiments humains, exactement comme sur la scène tragique antique, mais incarnée dans le monde moderne: la grande ville, le crime organisé, l'unité familiale et non plus politique, etc. Je continue de le penser, mais depuis quelques temps, j'ai aussi remarqué que cette scène-là devait beaucoup au XIXe, au romantisme et aux mythologies gothiques, à l'identification de la littérature avec la marge, avec le mal. J'ai notamment lu les travaux de Mario Praz qui trace des généalogies vraiment passionnantes de cette histoire. Et au fond, chez moi, ce XIXe siècle, c'est l'image que j'ai depuis l'enfance de la littérature, avec Edgar Poe et Conan Doyle. Dernier avantage du policier : il permet de travailler sur de petits espaces, et moi je crois que je me sens mieux dans une unité de lieu.

R.-M. A. - Selon Umberto Eco, "toute recherche a une structure policière ». 
T. V. - Oui, c'est sans doute vrai. Il y a toujours un suspens de la pensée, une enquête et une résolution. Même la philosophie la plus aride est toujours un drame. En fait, je suis complètement d'accord avec cette phrase.

R.-M. A. - Le recyclage des codes est un des éléments caractéristiques de la postmodernité : où vous situez-vous par rapport elle?

T. V. - Finalement je trouve cette idée de postmodernité de plus en plus juste. Non pas vraiment le recyclage des codes, mais ce sentiment que nous arrivons "après », que toutes les histoires ont été jouées et surtout déjouées, que nous appartenons à un monde de spectres, entièrement reconstitué, parce qu'entièrement détruit avant nous : peut-être il faudrait lire le $\mathrm{XX}^{\mathrm{e}}$ siècle comme ça, comme la mise à sac de trente siècles de littérature. Alors depuis quelques années, nous puiserions sur ce champ de ruines, dans lequel nous avons à disposition une sorte de capital global de figures à réassembler. Si la postmodernité peut ressembler à ça, alors oui, nous y sommes.

R.-M. A. - Ne s'agit-il pas aussi de sublimer les poncifs de la paralittérature par une écriture plus consciente d'elle-même, par un effort d'élévation poétique?

T. V. - Ce qui est vrai en tout cas, c'est que même dans cette grande mise à plat des hiérarchies et des figures, il y a toujours un moment où il faut recomposer des forces et des condensations: aussi l'effort d'élévation poétique est-ce surtout, dans le travail romanesque, l'effort pour charger les figures, pour les rendre actives et denses, que ce soit les paysages, les actions, les objets ou les personnages. C'est en fait 
un effort métonymique, où il faut faire rentrer beaucoup de contenu dans un contenant. Alors bien sûr, plus cet effort se fait, plus les images sont tendues ou intensifiées, et plus aussi l'écriture semble consciente d'elle-même : elle finit par se voir elle-même à l'œuvre.

R.-M. A. - Chez vous, il me semble que les tensions internes et les expansions de la syntaxe se substituent au suspense du récit et sont parties essentielles de la dynamique narrative. J'aimerais que vous réagissiez à la formule de Claude Simon sur ses problèmes d'écrivain : "commencer une phrase, puis la continuer, puis la terminer ».

T. V. - Je prends ce que vous dites pour un vrai compliment. En tout cas, c'est là qu'il me semble qu'il y a un effort de ma part, en ce qu'une phrase pour moi essaie toujours de résoudre une impression, une image, une sensation. Donc elle a son suspens, sa tension interne pour dynamiser et mêler les éléments, pour faire l'image, comme dirait Beckett - ce qui veut dire aussi faire la lumière, faire le cadre, faire l'arrièreplan, bref, un récit à part entière. Ensuite, il faudrait ajouter l'autre grande difficulté du récit pour moi, qui est la transition. Ce que vous appelez dynamique narrative, j'ai l'impression qu'elle doit provenir du glissement entre les phrases et plus encore entre les paragraphes. Bien sûr ce glissement peut procéder par rupture aussi bien que par contiguïté (et même quelquefois par anaphore), mais il doit avoir une efficace rythmique et produire une collusion chez le lecteur, et donc une continuité dynamique. Écrire du roman, pour moi, c'est créer des liens entre des éléments hétérogènes, c'est faire de l'homogène avec de l'hétérogène et pour ça, il n'y a qu'un outil, c'est la syntaxe. 
R.-M. A. - Puisque Simon a été longtemps associé à ce qu'il est convenu d'appeler le "Nouveau Roman» - auquel la figuration narrative est d'ailleurs en partie redevable - et que vous êtes publié par les Éditions de Minuit, la maison mère de la mouvance, j'aimerais savoir quelle importance vous accordez à cette tentative de rénovation romanesque, et dans l'histoire littéraire, et dans votre propre parcours de lecteur, puis d'écrivain.

T. V. - Dans l'histoire littéraire, à mon sens le Nouveau Roman synthétise l'après-guerre, en en ayant tous les enjeux formels mais aussi thématiques. Il y a un vrai dialogue avec le passé et aussi, quoi qu'on en dise, une lisibilité très forte. Si on compare avec Tel Quel, le Nouveau Roman n'a jamais abandonné la narration et le souci du récit, ni le souci du sens, et donc du sens commun. Même si certaines déclarations de Robbe-Grillet semblent un peu "déshumanisées", il y a toujours de la chair, du sensible et finalement l'ombre d'un référent. C'est ce qui les laisse bien vivants aujourd'hui, et c'est encore plus vrai pour Simon ou pour Duras. Quant à moi, dans mon propre parcours, c'est tout simplement cette littérature-là qui m'a donné un minimum de sens esthétique et a fait naître le sentiment que moi aussi je pouvais écrire. Je n'ai jamais trouvé littérature plus démocratique au fond que les narrateurs de Beckett, ou l'absence de narrateur chez Robbe-Grillet. C'est dans ce retrait-là que pour ma part j'ai pu trouver une place, en tant que lecteur d'abord, en tant que bénéficiaire d'un droit à l'écriture ensuite.

R.-M. A. - Vous êtes brestois, comme Robbe-Grillet. Pourriez-vous à la fois me donner votre opinion sur l'écrivain et me parler de l'empreinte éventuelle qu'a laissée votre ville natale 
dans votre cuvre, dans votre manière de voir le monde et d'aborder la littérature?

T. V. - Comme beaucoup de gens, je trouve que RobbeGrillet a trop parlé de son propre travail. En revanche, je suis un peu outré par la liquidation faite aussi vite et étayée à tort par l'antipathie du personnage. Les deux tiers de ses livres sont splendides, où pas une phrase, pas un adjectif ne respire autre chose que de la présence pure, littéraire et vivante. Le début de Dans le labyrinthe, j'aurais bien aimé l'avoir écrit. Je ne sais pas si on le réévaluera ou si toujours son aridité le rendra éloigné du «mainstream » de l'histoire littéraire, parce que je crois que c'est cette radicalité-là qui en fait aussi un écrivain très solitaire. Si on conserve le vieux critère de la singularité d'une œuvre, alors Robbe-Grillet est beaucoup plus grand, beaucoup plus seul que Claude Simon, qui doit décidément trop à Faulkner.

Pour ce qui est de Brest, c'est très vaste et très difficile à dire, parce qu'en un sens, j'y habite encore. Brest est devenu une fiction, un décor qui est lui-même mon territoire littéraire. J'ai l'impression d'avoir élu domicile là, de m'être sédentarisé dans un espace mental et visuel, avec cette ville très archétypale et la mer autour. Il y a dans ce type de "maquette" une simplicité géométrique proche de Kafka: territoire, frontière, limite, horizon, tout ça est fait d'angoisses autant que de projections rêvées, presque animales.

R.-M. A. - Le vent et la roche habitent votre ouvre: l'influence de la Bretagne originelle?

T. V. - Sûrement. Mais là aussi le réel de l'enfance a rencontré un territoire balisé littérairement, celui du roman 
anglo-saxon, puis du roman maritime. C'est une chance quand ses mythologies personnelles rencontrent des mythologies esthétiques, même si elles sont un peu anciennes...

R.-M. A. - Brest, c'est aussi le Finistère, une limite donc, géographique et symbolique. Or vos personnages sont également des marginaux, dans les franges de la représentation et de la société. À quoi correspond ce choix?

T. V. - Ce n'est pas vraiment un choix, plutôt un constat a posteriori qu'en effet les personnages sont un peu déroutés. Je n'ai pas beaucoup d'explication là-dessus, sinon que sans doute, derrière, se jouent une image de soi aussi bien qu'une image de l'art, que l'une comme l'autre, l'identité personnelle ou l'œuvre d'art ont du mal à être pensées au centre de l'espace sensible, économique ou politique, donc ni au centre du monde, ni au centre de la ville, ni dans des positions de force, mais plutôt, soit dans des positions de fatigue et de spectre, soit dans des positions de revanche ou même (je le ressens de plus en plus) d'attente et de reconquête. Brest comme antichambre des capitales.

R.-M. A. - Quand vous viviez à Tours, vous étiez dans le dénuement. En quoi cette expérience de vie a-t-elle influé sur votre pratique de la littérature? Il y a-t-il un lien avec le thème de l'absence - d'argent ou d'amour — dans plusieurs de vos livres?

T. V. - Pour un éventuel lien entre ma vie et mes livres, je ne saurais dire. Si l'absence y tient un grand rôle, j'ai envie de croire que c'est dans l'enfance que se forge ce genre de manque et d'obsession. Le dénuement dont vous parlez, un peu mythifié par d'autres, correspond plus simplement à une radicalité de vie. Il se trouve que je n'avais qu'une idée en tête - écrire - et 
que cette idée arrivait à m'arracher à tout, à me désincarner en quelque sorte. Et puis il y a une grande jouissance à n'avoir rien. C'est beaucoup plus excitant, même narcissiquement, que d'avoir « un peu ». Pour revenir à la littérature, il me semble que c'est aussi ce que cherchent mes personnages: un affranchissement qui passe par le vide, la destruction du passé, l'effacement des traces. Ils débutent souvent éclipsés ou absentés par les autres et peu à peu essaient d'aller vers leur propre présence, qui est leur affranchissement et qui passe souvent aussi par la liquidation des autres.

R.-M. A. - J'ai lu quelque part - je ne sais plus où - qu'on vous avait rapproché d'Olivier Rolin, à travers la figure $d u$ perdant magnifique. Ce dernier élément m'a paru pertinent, mais plutôt en le rapportant à Hemingway, Conrad et Melville. Qu'en dites-vous?

T. V. - Je n'ai pas lu Olivier Rolin mais pour les autres, le point commun, me semble-t-il, c'est que ce sont des œuvres très initiatiques. En tout cas, c'est comme ça que je lis Melville et Conrad, comme des romans de formation et des méditations sur l'existence. Et cette méditation est d'autant plus possible que les narrateurs ont été meurtris, qu'ils font souvent le récit d'une expérience violente, complexe et qui les a grandis, même dans la défaite. D'où peut-être cette expression trop employée de «perdant magnifique ».

R.-M. A. - Il y a une dimension crépusculaire dans vos livres. Votre art serait-il donc tourné vers le couchant?

T. V. - Je ne sais pas. J'aime beaucoup m'appuyer sur la fatigue pour commencer mon travail, c'est une manière de faire 
retomber un peu la tension inhérente au geste d'écrire, et cette fatigue se figure peut-être mieux dans le couchant.

R.-M. A. - L'Absolue perfection du crime repose sur la trahison; Insoupçonnables est porté par le thème du faux frère; dans Vers la fiction vraie, vous écrivez qu'il y a forcément trahison de la lettre. Qu'est-ce ce qui vous touche dans ce dénominateur commun?

T. V. - Je me demande si je suis sensible à la trahison en tant que telle, ou si elle est simplement le lieu épique de la déception. Je veux dire que ce qui me touche, c'est l'écart entre l'espoir posé dans les choses et les êtres, et la réponse des choses et des êtres à cet espoir. La trahison est simplement la situation superlative de cette déception, qui n'est elle-même que le dégonflement de la baudruche, c'est-à-dire le dégonflement des projections inconsidérées faites sur le monde.

R.-M. A. - Seriez-vous pessimiste?

T. V. - En littérature, oui, pourquoi pas. En tout cas, je ne trouve pas très excitant de crier au bonheur dans l'écriture : le bonheur, ce serait plutôt la promesse extérieure au livre, la vie vécue peut-être, mais l'écriture, c'est différent, c'est une longue déploration, un échec préparé et qui jouit de lui-même. Ce peut être très heureux, l'écriture, mais forcément sur des cendres, sur quelque chose de noir au fond.

R.-M. A. - Et la tragédie dans tout cela? N'est-elle pas au cour de vos préoccupations?

T. V. - Disons qu'elle est une ombre derrière l'épaule... 
R.-M. A. - «À grand manque grande invention. ", résume Vers la fiction vraie, à propos de la mélancolie. La fiction, selon vous, serait un antidote à la solitude et au néant: "C'est ainsi, à proportion de sa peur, à proportion du gouffre dont il réchappe, que le mélancolique engendre le monde, soit-il tout gonflé de manque. »C'est un autoportrait?

T. V. - Ce que je sais de cela, c'est qu'à un moment j'ai eu besoin de regarder toutes ces choses psychiques de face, notamment tous les empêcheurs d'écrire, et à force de réfléchir dessus, je suis arrivé à ce qui est comme une tarte à la crème finalement: la mélancolie. Au fond, ce travail autour de la mélancolie était une sorte de développement de la question d'Aristote dans le Problème XXX. C'était vraiment ça qui m'intéressait. Et du coup j'arrivais à cette conclusion simple : quand on est mélancolique, ou bien on agit dans le monde mais on passe pour fou, ou bien on choisit le monde des fictions et on est à sa place. En ce sens, peut-être, c'est un autoportrait.

R.-M. A. - Serait-ce dans le même ordre d'idées que vous vous intéressez aux romans et aux films noirs?

T. V. - Je ne sais pas, parce qu'on ne peut pas dire que le noir soit la seule réponse à la mélancolie. Ce que je crois savoir, c'est que la fiction policière ou noire met forcément en scène des êtres débordants, qui en font trop, et donc vont engendrer des conflits, soit avec la justice, le monde, soit avec eux-mêmes. Mais dire cela, c'est sans doute ne rien dire, parce que ce n'est pas une spécificité du noir : quel genre de fiction ne mettrait pas en scène des êtres débordants?

R.-M. A. - Dans la citation ci-dessus, il y a deux formules récurrentes sous votre plume d'essayiste: «à proportion» et 
« soit-il ». Effet de style? Tic d'écriture ? Souci du volume? Vou d'absolution en forme d'hypothèse?

T. V. - Tic d'écriture sans doute, mais c'est surtout que ma plume d'essayiste, pour reprendre votre expression, est fragile. Pour tout vous dire, je me suis essayé à ce genre-là d'abord pour régler des comptes personnels avec certains points théoriques. Mais je suis obligé de constater, avec un peu de recul, qu'il y a une différence entre vouloir se mettre au clair avec une question, en l'occurrence la mélancolie, et apporter une contribution au champ du savoir.

R.-M. A. - Ladite citation est incluse dans un texte où Flaubert tient la première place, mais elle m'a tout d'abord fait penser à Robert Pinget. L'avez-vous lu?

T. V. - Moins que Beckett, s'il faut les comparer. Mais j'ai une tendresse très forte pour ce que j'ai lu, notamment Quelqu'un et puis les pensées de Monsieur Songe. Je pense à cette phrase qui me hante par sa violence et sa justesse, dans Taches d'encre, je crois : "Il ne faut pas confondre ses goûts et ses talents. » Il y a cette sagesse noire chez Pinget, qui le rend dur quelquefois mais nous rend curieusement tendres à son égard, parce qu'il semble d'abord dur avec lui-même.

R.-M. A. - Robert éprouvait une grande admiration pour Cervantès - comme vous, je crois. L'écart entre un idéal de grande élévation et la fatale déception du quotidien, qu'on trouve également chez Dostoïevski, pourriez-vous m'en parler?

T. V. - Oui. Je peux essayer. D'abord je vous remercie de votre question parce que je n'aurais pas osé rapprocher moimême Cervantès et Dostoïevski sur ce terrain, et pourtant je me rends compte que c'est ce qui me les rend plus grands que tous 
les autres: cette manière de se débattre avec une pensée qu'aucun corps, aucune incarnation, ne semble capable de loger. Et surtout pas bien sûr, le corps de la lettre. Pour tous ces personnages, il y a cela, une pensée hypertrophiée qui prend le pas sur le réel jusqu'à la déception ou la folie : c'est drôle chez Cervantès, c'est plus tragique chez Dostoïevski. Mais peut-être la différence, si je peux me permettre, avec mon travail, c'est que mes narrateurs, au moment où ils racontent, ont enfin trouvé un espace de conciliation entre leurs pensées, leurs rêves ou leurs idéaux, et la réalité de leur quotidien. C'est même cette conciliation qui permet qu'un récit soit possible, sans quoi ils seraient encore brûlés par l'espoir de leur folie, ou par la violence de leur déception. Peut-être même que le récit qu'ils font, ces narrateurs, c'est le récit du passage de leur réconciliation, dût-il bien sûr être le récit d'un crime ou d'un effacement des traces. Mais vous m'obligez là à penser des choses que je n'ai jamais pensées avant.

R.-M. A. - L'île du mélancolique est sans cesse menacée d'engloutissement, dites-vous : telle l'île mystérieuse du capitaine Nemo, de personne, du non-sujet?

T. V. - Oui, je crois. C'est un des seuls passages de mon travail théorique que je peux reprendre aujourd'hui sans trop de gêne, à propos du tourbillon chez Poe et du trou noir en général. Il me semble que l'identité est une négociation de chaque instant avec les puissances du vide, mais qu'en même temps il s'agit de continuer à négocier pour ne pas sombrer.

R.-M. A. - Saviez-vous que le nom de Fernando Pessoa signifie justement «personne»? 
T. V. - Oui, je n'y croyais pas quand on me l'a dit. C'était trop beau pour être vrai, tellement ce semble magique. En tout cas Pessoa fait partie de ceux qui m'accompagnent et d'une manière d'autant plus fréquente que chaque hétéronyme représente un peu de chaque possible littéraire. Quand on ne se sent plus Alvaro de Campos, alors on se sent Alberto Caeiro.

R.-M. A. - Et la saudade, ça vous touche?

T. V. - Qui répondrait non ? En même temps : qui pourrait s'arroger un oui qui appartient à tout le monde ? Bien sûr ça me touche, mais c'est peut-être ce qui touche qu'on pourrait appeler ainsi.

R.-M. A. - À quand la couleur dans vos livres?

T. V. - C'est vrai qu'on me dit souvent que mes livres ont l'air d'être en noir et blanc. Je suppose que cela tient à l'imaginaire cinématographique qu'ils font naître chez les lecteurs. Mais pour ma part, je tiens à le dire, j'écris en couleurs : par exemple, Le Black Note est un livre jaune et noir, L'Absolue perfection $d u$ crime est un livre rouge et noir, enfin Insoupçonnables est un livre avec beaucoup de bleu et de jaune (et aussi du vert sur le terrain de golf). Je n'ai jamais cherché à recenser les adjectifs de couleurs dans mes livres, mais je suis sûr qu'il y en a plus qu'on ne croit.

R.-M. A. - Quels sont vos projets actuels?

T. V. - Je viens de terminer un roman qui sort en janvier prochain et qui s'appellera Paris-Brest. C'est ce qu'on peut appeler un roman familial, en ce sens plutôt différent des précédents, sauf à considérer (ce que je crois) que le roman policier était une bonne manière de faire des romans familiaux sans que ça se voie trop. Par ailleurs, en ce moment je m'amuse 
beaucoup en produisant un feuilleton, sur commande, pour un centre culturel qui le diffuse sur papier. Cette contrainte est curieusement stimulante, surtout le fait de devoir écrire vite et donner vite ce qu'on a écrit, sans trop regarder derrière. C'est décomplexant d'un point de vue narratif, et c'est ce qu'il me faut.

Dimanche 2 novembre 2008

\section{Bibliographie}

ColleCTIF. 2008, Figuration narrative, Paris, catalogue RMNCentre Pompidou, 350 p., 450 ill.

PINGET, Robert. 1965, Quelqu'un, Paris, Minuit;

—. 1982, Monsieur Songe, Paris, Minuit;

—. 1997, Taches d'encre, Paris, Minuit.

RoBBE-GRILLET, Alain. 1959, Dans le labyrinthe, Paris, Minuit.

VIEL, Tanguy. 1998, Le Black Note, Paris, Minuit;

—. 1999, Cinéma, Paris, Minuit;

—. 2001, L'Absolue perfection du crime, Paris, Minuit;

-. 2005, Vers la fiction vraie, http://www.inventaireinvention.com/melancolies/viel partie3.htm

—. 2008, Insoupçonnables, Paris, Minuit. 